\title{
A far-field based T-matrix method for two dimensional obstacle scattering
}

\author{
M. Ganesh ${ }^{1} \quad$ S. C. Hawkins ${ }^{2}$
}

(Received 13 January 2010; revised 6 April 2010)

\begin{abstract}
The infinite T-matrix completely describes the acoustic scattering properties of an obstacle. The T-matrix is extremely important for many applications because it is computationally cheap to use the $\mathrm{T}$ matrix to simulate scattering for many different orientations of the obstacle or incident waves. The T-matrix is usually computed using the null field method. However, the null field method is often numerically unstable for acoustically large obstacles or for obstacles with large aspect ratios. We describe an efficient and stable method for computing the T-matrix for sound-soft, sound-hard, absorbing, and dielectric scattering, using numerical far field values obtained using any method. We demonstrate the algorithm by generalizing the recently proposed fundamental solution and non-polynomial finite element methods for the far field and T-matrix computations.
\end{abstract}

http://anziamj . austms.org.au/ojs/index.php/ANZIAMJ/article/view/2581 gives this article, (c) Austral. Mathematical Soc. 2010. Published May 12, 2010. ISSN 1446-8735. (Print two pages per sheet of paper.) 


\section{Contents}

1 Introduction

C216

2 Expansions of the acoustic field

C218

3 Far field based T-matrix computation

C220

4 Symmetry properties of the T-matrix

C222

5 Numerical experiments

C225

References

$\mathrm{C} 227$

\section{Introduction}

Wave scattering simulations involve computing the acoustic/electromagnetic field generated when a known incident field interacts with an obstacle. The Tmatrix method is based on the fact that both the incident and scattered fields possess series expansions in terms of wave functions, and the coefficients in the series are connected by an infinite matrix known as the T-matrix [3, 6, 7].

Applications typically use a truncated approximation to the infinite T-matrix. The truncated T-matrix method is extremely useful in applications such as multiple scattering because the individual T-matrices of each individual scatterer are efficiently combined using the translation-addition theorem [6, 7]. The T-matrix is essential in applications requiring averaged scattering properties over a range of incident directions or orientations, because such averages are computed directly from the T-matrix.

The T-matrix was developed initially for electromagnetic scattering by Waterman [8]. The truncated T-matrix is usually computed using the null field method $[3,6,8]$. For medium to high frequency problems or high aspect ratio 
obstacles the null field method is numerically unstable due to fast growth of the Hankel functions used in the null field method [6].

Recently, alternative algorithms based on far field computations have been developed for three dimension electromagnetic [5] and acoustic scattering [4]. These algorithms replace the spherical Hankel functions by their far fields, which do not exhibit fast growth, leading to numerically stable algorithms. These algorithms critically depend on the availability of fast surface integral algorithms for numerical far field computations.

We describe a new, numerically stable, far field based algorithm for computing the T-matrix for two dimensional acoustic/dielectric scattering. We focus on the problem of how to use numerically computed far field values (obtained using any method) to compute the T-matrix for sound-soft, sound-hard, absorbing, and dielectric obstacles. In this work, for far field computations, we use the method of fundamental solution in contrast to the surface integral equation techniques $[5,4]$. The advantage of this method is that it is applicable to a range of boundary conditions, and to scattering by polygons by the addition of non-polynomial finite elements [1].

The next two sections develop all of the details required for the T-matrix computations. Section 4 proves a symmetry property of the acoustic Tmatrix that can be used to measure the quality of a numerically computed truncated T-matrix. Section 5 computationally demonstrates our acoustic T-matrix computations for smooth and polygonal obstacles with a range of boundary conditions, including a transmission (TM-polarized dielectric scattering) problem for a penetrable scatterer. 


\section{Expansions of the acoustic field}

The time-harmonic radiating acoustic field $u$ scattered by a two dimensional scatterer D in a homogeneous medium satisfies the Helmholtz equation

$$
\triangle \mathfrak{u}(\boldsymbol{x})+k^{2} u(x)=0, \quad x \in \mathbb{R}^{2} \backslash \overline{\mathrm{D}},
$$

where $k=2 \pi / \lambda$ is the wavenumber and $\lambda$ is the wavelength, and the Sommerfeld radiation condition

$$
\lim _{|x| \rightarrow \infty} \sqrt{|x|}\left(\frac{\partial u}{\partial x}-i k u\right)=0,
$$

where the limit holds uniformly in all directions $\widehat{x}=x /|x|$. The scattered field $u$ is induced by the incident field $u^{i}$ with wavenumber $k$ via one of the following boundary conditions (properties of the scatterer)

$$
\begin{aligned}
\mathrm{U}(\boldsymbol{x}) & =0, & \boldsymbol{x} \in \partial \mathrm{D}, & \\
\frac{\mathrm{dU}}{\mathrm{dn}}(\boldsymbol{x}) & =0, \quad \boldsymbol{x} \in \partial \mathrm{D}, & & \text { (sound-soft) } \\
\mathrm{U}(\boldsymbol{x})+\lambda \frac{\mathrm{dU}}{\mathrm{dn}}(\boldsymbol{x}) & =0, \quad \lambda \in \mathbb{R}, \boldsymbol{x} \in \partial \mathrm{D}, & & \text { (absorbing) }
\end{aligned}
$$

where $\mathrm{U}=\mathfrak{u}^{\mathrm{i}}+\mathfrak{u}$ is the total field, or via the transmission (TM-polarized dielectric) boundary conditions

$$
\mathrm{U}(\boldsymbol{x})=\mathrm{V}(\boldsymbol{x}), \quad \frac{\mathrm{dU}}{\mathrm{d} \mathfrak{n}}(\boldsymbol{x})=\frac{\mathrm{dV}}{\mathrm{d} \mathfrak{n}}(\boldsymbol{x}), \quad \boldsymbol{x} \in \partial \mathrm{D}, \quad \text { (penetrable) }
$$

where $\mathbf{U}(\boldsymbol{x})=\mathfrak{u}^{\mathrm{i}}(\boldsymbol{x})+\mathfrak{u}(\boldsymbol{x})$ is the total exterior field for $\boldsymbol{x} \in \mathbb{R}^{2} \backslash \mathrm{D}$ and $\mathrm{V}(\boldsymbol{x})$ is the interior field for $\boldsymbol{x} \in \mathrm{D}$, satisfying the interior Helmholtz equation in $\mathrm{D}$ with wavenumber $k_{\text {int }}$. Hence for transmission (or dielectric) scattering, the refractive index (or dielectric constant) is $k / k_{\text {int }}$ (or $k^{2} / k_{\text {int }}^{2}$ ).

The T-matrix connects expansions of the incident field and scattered field in terms of wave functions. In particular, the basic tools for the expansions are 
the regular wave functions

$$
\widetilde{e}_{n}(\boldsymbol{x})=\mathrm{J}_{|\mathrm{n}|}(\mathrm{k}|\boldsymbol{x}|) \exp (\mathrm{in} \theta), \quad \theta=\theta(\boldsymbol{x}),
$$

and radiating wave functions

$$
e_{\mathfrak{n}}(\boldsymbol{x})=\mathrm{H}_{|\mathrm{n}|}^{(1)}(\mathrm{k}|\boldsymbol{x}|) \exp (\mathrm{in} \theta), \quad \theta=\theta(\boldsymbol{x}),
$$

where $J_{n}$ is the first kind Bessel function of degree $n$, and $H_{n}^{(1)}$ is the first kind Hankel function of degree $n$. Here we use that the point $\boldsymbol{x}$ has a unique representation in polar coordinates with radius $|\boldsymbol{x}|$ and azimuth $\theta$.

Both kinds of wave functions satisfy the Helmholtz equation (1). The radiating wave functions additionally satisfy the radiation condition (2), a constraint on the scattered acoustic fields that guarantees their outgoing nature. The T-matrix is obtained by expanding the incident and scattered fields as

$$
u^{i}(x)=\sum_{n=-\infty}^{\infty} p_{n} \widetilde{e}_{n}(x), \quad u(x)=\sum_{n=-\infty}^{\infty} a_{n} e_{n}(x) .
$$

Typically the coefficients $p_{n}$ of the incident field are known. For example, these coefficients are given analytically when $u^{i}$ is a plane wave. Since the Helmholtz equation (1) (which connects the incident and scattered fields) is linear, there exist coefficients $t_{\mathfrak{n}^{\prime}, n}$ such that

$$
a_{n^{\prime}}=\sum_{n=-\infty}^{\infty} t_{n^{\prime}, n} p_{n}
$$

Writing $\boldsymbol{a}=\left(a_{n}\right)$ and $\boldsymbol{p}=\left(p_{n}\right)$, and denoting $T$ for the T-matrix, we have

$$
\mathbf{a}=\mathrm{Tp}, \quad \mathrm{T}=\left[\mathrm{t}_{\mathrm{n}^{\prime}, \mathrm{n}}\right] .
$$

Using (10) the coefficients in the expansion of the scattered field are computed easily (for any incident direction) by matrix vector multiplication, if an appropriately truncated version (with high order accuracy) of the T-matrix (associated with D) is computed and stored. 


\section{$3 \quad$ Far field based T-matrix computation}

A given radiating acoustic field $u$ has far field $\boldsymbol{u}^{\infty}$ and acoustic cross section (ACS) $\epsilon^{\mathrm{dB}}$ (measured in decibels), defined by

$$
u^{\infty}(\widehat{x})=\lim _{|x| \rightarrow \infty} \sqrt{|x|} e^{-i k|x|} u(x), \quad \epsilon^{\mathrm{dB}}(\widehat{\boldsymbol{x}} ; \widehat{\mathbf{d}})=10 \log _{10}\left(2 \pi\left|\mathbf{u}^{\infty}(\widehat{\boldsymbol{x}})\right|^{2}\right),
$$

which describes the asymptotic behaviour of the exterior field at large distances from the scatterer. Here $\widehat{\mathbf{d}}$ is the direction of the incident field and the resulting far field is measured in the direction $\widehat{x}=\boldsymbol{x} /|\boldsymbol{x}|$.

For backscattered ACS, $\widehat{\boldsymbol{x}}=-\widehat{\mathbf{d}}$. The backscattered ACS of the scatterer as a function of $\widehat{\boldsymbol{x}}$ (and hence $\widehat{\mathbf{d}}$ ) around D is known as the monostatic ACS. To describe the monostatic ACS of a scatterer, the exterior Helmholtz problem needs to be solved with thousands of incident directions. For monostatic ACS, T-matrix is an extremely powerful tool. For bistatic ACS, the incident direction $\widehat{\mathbf{d}}$ is fixed and the resulting field is measured around the obstacle.

Let $S u^{i}$ denote the scattered field induced by the incident field $\mathfrak{u}^{i}$ through one of the the boundary conditions (3)-(6). Applying the operator $\mathbf{S}$ to the series representation (9) for the incident field we get

$$
u=S u^{i}=S \sum_{n=-\infty}^{\infty} p_{n} \widetilde{e}_{n}=\sum_{n=-\infty}^{\infty} p_{n} S \widetilde{e}_{n}
$$

where each $S \widetilde{e}_{n}$ denotes the scattered field induced by $\widetilde{e}_{n}$. Each $S \widetilde{e}_{n}$ is a radiating field that satisfies the Helmholtz equation (1) and so has an expansion (with some coefficients $t_{n^{\prime}, n}$ ) in the radiating spherical wave functions

$$
\mathrm{S} \widetilde{e}_{\mathrm{n}}=\sum_{n^{\prime}=-\infty}^{\infty} t_{n^{\prime}, n} e_{n^{\prime}}, \quad n=-\infty, \ldots, \infty .
$$

Using (11), and the limit as $r \rightarrow \infty$ of the Hankel functions $H_{n}^{(1)}(r)$, the far 
field $e_{n^{\prime}}^{\infty}$ of the radiating spherical wave function $e_{n^{\prime}}$ is

$$
e_{n^{\prime}}^{\infty}(\theta)=\sqrt{\frac{1}{\pi k}}(-\mathfrak{i})^{\left|n^{\prime}\right|}(1-\mathfrak{i}) \exp \left(\mathfrak{i n}^{\prime} \theta\right)
$$

Using (13) and (14), we derive for $\widetilde{S}_{\mathfrak{n}}$ the far field

$$
S^{\infty} \widetilde{\boldsymbol{e}}_{\mathfrak{n}}=\sum_{n^{\prime}=-\infty}^{\infty} t_{\mathfrak{n}^{\prime}, n} \sqrt{\frac{1}{\pi k}}(-i)^{\left|\mathfrak{n}^{\prime}\right|}(1-i) \exp \left(\boldsymbol{i n}^{\prime} \theta\right) .
$$

The key to our algorithm is that we compute an accurate approximation to $S^{\infty} \widetilde{\boldsymbol{e}}_{\mathrm{n}}$ using some appropriate numerical technique. Thus in (15) only the T-matrix entries $t_{\mathfrak{n}^{\prime}, \mathfrak{n}}$ are unknown. Using the orthogonality of $\exp \left(\mathrm{in}^{\prime} \theta\right)$, we pick out the T-matrix entries from a numerically computed approximation to $S^{\infty} \widetilde{e}_{n}$ by taking the inner product

$$
t_{n^{\prime}, n}=\frac{1}{4} \sqrt{\frac{k}{\pi}} i^{\left|n^{\prime}\right|}(1+i)\left\langle S^{\infty} \widetilde{e}_{\mathfrak{n}}, \exp \left(\mathfrak{i n}^{\prime} \theta\right)\right\rangle,
$$

where $\langle\cdot, \cdot\rangle$ is the usual inner product on the unit circle.

For practical computations the infinite T-matrix must be truncated, so that we restrict to wave functions of order $n, n^{\prime}=-N, \ldots, N$, in all expansions. That is, we replace the symbol $\infty$ in all of the above infinite sums with a parameter $\mathrm{N}$. In practice we evaluate the inner product (16) using a Gauss quadrature rule of order $2 \mathrm{~N}+2$. This quadrature scheme converges superalgebraically for smooth functions on the circle such as those in (16).

Using (10) and (16), to compute the T-matrix we must perform one far field computation to compute, using any method, an approximation to the far field $S^{\infty} \widetilde{e}_{n}$ for each incident wave function $\widetilde{e}_{n}, n=-N, \ldots, N$. That is, for a chosen truncation parameter $\mathbf{N}$, we compute solutions of (1)-(6) with $u^{i}=\widetilde{e}_{n}$, for $n=-N, \ldots, N$. For the numerical experiments in Section 5 we compute the far fields using the method of fundamental solution (MFS) in the case of smooth boundaries $\partial \mathrm{D}$, and MFS combined with a non-polynomial 
finite element method for polygonal boundaries $\partial \mathrm{D}$. Our implementation is based on a new T-matrix class which extends MPSPACK [1]. The high order convergence of the MFs solution leads to small linear least squares problems that we solve very efficiently using QR factorisation, even for several right hand sides induced by the many incident wave functions.

\section{Symmetry properties of the T-matrix}

The accuracy of a numerically computed T-matrix is usually measured using symmetry properties. A symmetry property similar to the one proved below is known for three dimensional sound-soft acoustic scattering [6, Theorem 7.4]. To our knowledge (and based on discussions with Martin [6]) the following two dimensional counterpart is new, even for the sound-soft case.

Theorem 1 Consider the scattering problem for an obstacle D satisfying one of the boundary conditions (3)-(6). Then the infinite T-matrix satisfies

$$
\mathrm{T}+\mathrm{T}^{*}+2 \mathrm{~T}^{*} \mathrm{~T}=0
$$

where $\mathrm{T}^{*}$ denotes the conjugate transpose of $\mathrm{T}$.

Proof: Let $\Omega$ be a disk with circular boundary $\partial \Omega$, with radius a circumscribing the scatterer $\mathrm{D}$, and for fields $\mathrm{U}, \mathrm{V}$ on $\Omega$, define the sesquilinear functional

$$
[\mathrm{u}, \mathrm{v}]=\int_{\partial \Omega}\left(\mathrm{u} \frac{\partial \bar{V}}{\partial \mathfrak{n}}-\bar{v} \frac{\partial \mathrm{U}}{\partial \mathfrak{n}}\right) \mathrm{dS} .
$$

Using the Wronskian for the Bessel and Hankel functions [2, Equation (3.56)] we have

$$
\left[\widetilde{e}_{n}, \widetilde{e}_{n^{\prime}}\right]=0, \quad\left[\widetilde{e}_{n}, e_{n^{\prime}}\right]=\left[e_{n}, \widetilde{e}_{n^{\prime}}\right]=-4 i \delta_{n, n^{\prime}}, \quad\left[e_{n}, e_{n^{\prime}}\right]=-8 i \delta_{n, n^{\prime}} .
$$


Let $\mathrm{U}$ and $\widetilde{\mathrm{U}}$ be total exterior field solutions of (1) with incident and scattered fields satisfying one of the boundary conditions (3)-(6). Using Green's theorem in the region between $\partial \mathrm{D}$ and $\partial \Omega$ and (1),

$$
\begin{aligned}
{[u, \widetilde{u}] } & =\int_{\partial \Omega}\left(u \frac{\partial \widetilde{\widetilde{u}}}{\partial \mathfrak{n}}-\overline{\widetilde{u}} \frac{\partial u}{\partial \mathfrak{n}}\right) d S \\
& =\int_{\Omega \backslash D}(u \triangle \overline{\widetilde{u}}-\overline{\widetilde{u}} \Delta u) d s+\int_{\partial D}\left(u \frac{\partial \overline{\widetilde{u}}}{\partial \mathfrak{n}}-\overline{\widetilde{u}} \frac{\partial u}{\partial \mathfrak{n}}\right) d S \\
& =\int_{\partial D}\left(u \frac{\partial \widetilde{u}}{\partial \mathfrak{n}}-\widetilde{\widetilde{u}} \frac{\partial u}{\partial \mathfrak{n}}\right) d S .
\end{aligned}
$$

If $\mathrm{U}$ and $\widetilde{\mathrm{U}}$ satisfy the Dirichlet boundary condition (3) or the Neumann boundary condition (4), then the integrand of the boundary integral in (19) is zero and hence $[\mathrm{U}, \widetilde{\mathrm{U}}]=0$.

If $\mathrm{U}$ and $\widetilde{\mathrm{U}}$ satisfy the Robin boundary condition (5), then

$$
u \frac{\partial \widetilde{\widetilde{u}}}{\partial n}-\overline{\widetilde{u}} \frac{\partial u}{\partial n}=-\lambda \frac{\partial u}{\partial n} \frac{\partial \widetilde{\widetilde{u}}}{\partial n}+\bar{\lambda} \frac{\partial \widetilde{\widetilde{u}}}{\partial \mathfrak{n}} \frac{\partial u}{\partial n}=(\bar{\lambda}-\lambda) \frac{\partial u}{\partial n} \frac{\partial \widetilde{\widetilde{u}}}{\partial n} .
$$

Since $\lambda \in \mathbb{R}$ in (5), we have $\bar{\lambda}-\lambda=0$ and hence, in this case also, $[\mathrm{U}, \widetilde{\mathrm{U}}]=0$. On the other hand, suppose that $\mathrm{U}$ and $\widetilde{\mathrm{U}}$ satisfy the transmission boundary condition (6) with corresponding interior fields $V$ and $\widetilde{V}$. Then using (6), (19), Green's theorem inside D, and that $\mathrm{V}$ and $\widetilde{V}$ satisfy the Helmholtz equation (1) inside D,

$$
\begin{aligned}
{[\mathrm{U}, \widetilde{\mathrm{U}}]=\int_{\partial \mathrm{D}}\left(\mathrm{u} \frac{\partial \overline{\widetilde{U}}}{\partial \mathrm{n}}-\overline{\widetilde{\mathrm{U}}} \frac{\partial \mathrm{U}}{\partial \mathrm{n}}\right) \mathrm{dS} } & =\int_{\partial \mathrm{D}}\left(\mathrm{v} \frac{\partial \overline{\widetilde{V}}}{\partial \mathrm{n}}-\overline{\widetilde{V}} \frac{\partial \mathrm{V}}{\partial \mathrm{n}}\right) \mathrm{dS} \\
& =\int_{D}(\mathrm{v} \triangle \overline{\widetilde{V}}-\overline{\widetilde{V}} \triangle \mathrm{V}) \mathrm{ds}=0
\end{aligned}
$$


Thus, for all boundary conditions (3)-(6) (with $\lambda \in \mathbb{R}$ for the Robin boundary condition) we have established $[\mathrm{U}, \widetilde{\mathrm{U}}]=0$.

Expanding $\mathfrak{u}^{\mathrm{i}}$ and $\widetilde{\boldsymbol{u}}^{\mathrm{i}}$ in terms of the entire wave functions and $\boldsymbol{u}$ and $\widetilde{\boldsymbol{u}}$ in terms of the radiating wave functions,

$\mathrm{u}(\boldsymbol{x})=\sum_{\mathrm{n}=-\infty}^{\infty}\left(\mathrm{d}_{\mathrm{n}} \widetilde{\mathrm{e}}_{\mathrm{n}}(\boldsymbol{x})+\mathrm{c}_{\mathrm{n}} \mathrm{e}_{\mathrm{n}}(\boldsymbol{x})\right), \quad \widetilde{\mathrm{u}}(\boldsymbol{x})=\sum_{n^{\prime}=-\infty}^{\infty}\left(\widetilde{\mathrm{d}}_{\mathrm{n}^{\prime}} \widetilde{\mathrm{e}}_{\mathrm{n}^{\prime}}(\boldsymbol{x})+\widetilde{\mathrm{c}}_{\mathrm{n}^{\prime}} \mathrm{e}_{\mathrm{n}^{\prime}}(\boldsymbol{x})\right)$, and using the T-matrix

$$
c_{\mathfrak{n}}=\sum_{m=-\infty}^{\infty} \mathrm{T}_{\mathrm{nm}} \mathrm{d}_{\mathrm{m}}, \quad \widetilde{\mathbf{c}}_{\mathfrak{n}^{\prime}}=\sum_{\mathrm{m}^{\prime}=-\infty}^{\infty} \mathrm{T}_{\mathrm{n}^{\prime} \mathrm{m}^{\prime}} \widetilde{\mathrm{d}}_{\mathrm{m}^{\prime}} .
$$

Using these expansions, together with sesquilinearity of $[\cdot, \cdot],(18)$ and (20),

$$
\begin{aligned}
& {[\mathrm{u}, \widetilde{\mathrm{u}}]=\left[\sum_{n=-\infty}^{\infty}\left(\mathrm{d}_{\mathrm{n}} \widetilde{\mathrm{e}}_{\mathrm{n}}+\mathrm{c}_{\mathrm{n}} \mathrm{e}_{\mathrm{n}}\right), \sum_{\mathrm{n}^{\prime}=-\infty}^{\infty}\left(\widetilde{\mathrm{d}}_{\mathrm{n}^{\prime}} \widetilde{\mathrm{e}}_{\mathrm{n}^{\prime}}+\widetilde{\mathrm{c}}_{\mathrm{n}^{\prime}} \mathrm{e}_{\mathrm{n}^{\prime}}\right)\right]} \\
& =\sum_{n=-\infty}^{\infty} \sum_{n^{\prime}=-\infty}^{\infty}\left(d_{n} \overline{\widetilde{d}_{n^{\prime}}}\left[\widetilde{\boldsymbol{e}}_{n}, \widetilde{\boldsymbol{e}}_{n^{\prime}}\right]+d_{n} \overline{\widetilde{\boldsymbol{c}}_{n^{\prime}}}\left[\widetilde{\boldsymbol{e}}_{n}, e_{n^{\prime}}\right]\right. \\
& \left.+c_{n} \overline{\widetilde{d}_{n^{\prime}}}\left[e_{n}, \widetilde{e}_{n^{\prime}}\right]+c_{n} \overline{\widetilde{c}_{n^{\prime}}}\left[e_{n}, e_{n^{\prime}}\right]\right) \\
& =\sum_{n=-\infty}^{\infty}-4 i\left(d_{n} \overline{\widetilde{c}_{n}}+c_{n} \overline{\widetilde{d}_{n}}+2 c_{n} \overline{\widetilde{c}_{n}}\right) \\
& =-4 i\left\{\sum_{n=-\infty}^{\infty} \sum_{m=-\infty}^{\infty} \overline{\mathrm{T}_{n m}} \mathrm{~d}_{\mathrm{n}} \overline{\widetilde{\mathrm{d}}_{\mathrm{m}}}+\sum_{\mathrm{m}=-\infty}^{\infty} \sum_{n=-\infty}^{\infty} \mathrm{T}_{m n} \mathrm{~d}_{\mathrm{n}} \overline{\widetilde{\mathrm{d}}_{\mathrm{m}}}\right. \\
& \left.+2 \sum_{m^{\prime}=-\infty}^{\infty} \sum_{n=-\infty}^{\infty} \sum_{m=-\infty}^{\infty} T_{m^{\prime} n} \overline{T_{m^{\prime} m}} d_{n} \overline{\tilde{d}_{m}}\right\} \\
& =-4 i \sum_{n=-\infty}^{\infty} \sum_{m=-\infty}^{\infty}\left(\overline{T_{n m}}+T_{m n}+2 \sum_{m^{\prime}=-\infty}^{\infty} T_{m^{\prime} n} \overline{T_{m^{\prime} m}}\right) d_{n} \overline{\widetilde{d}_{m}} .
\end{aligned}
$$


This must hold for any incident fields $\mathfrak{u}^{\mathrm{i}}$ and $\widetilde{\mathfrak{u}}^{\mathrm{i}}$ and so using $[\mathrm{u}, \widetilde{\mathrm{u}}]=0$, we get

$$
\overline{T_{n m}}+T_{m n}+2 \sum_{m^{\prime}=-\infty}^{\infty} T_{m^{\prime} n} \overline{T_{m^{\prime} m}}=0, \quad n, m=-\infty, \ldots, \infty .
$$

That is,

$$
\left(T^{*}+T+2 T^{*} T\right)_{m n}=0, \quad n, m=-\infty, \ldots, \infty .
$$

\section{$5 \quad$ Numerical experiments}

We demonstrate the convergence and accuracy of our algorithm using the symmetry property in Theorem 1 for smooth and polygonal scatterers with a range of boundary conditions. We visually demonstrate the algorithm with acoustic cross section computations, using our computed T-matrix.

The exact infinite T-matrix satisfies (17). The equality to zero in (17) does not hold when the infinite T-matrix is replaced by its numerically computed and truncated approximation. In this case, the deviation from zero of the right hand side is useful as a measure of the error in the approximate Tmatrix. In particular, we define

$$
\operatorname{Err}(\mathrm{N}):=\max _{\mathrm{n}=-\mathrm{N}, \ldots, \mathrm{N}} \max _{n^{\prime}=-\mathrm{N}, \ldots, \mathrm{N}}\left|\left(\mathrm{T}^{*}+\mathrm{T}+2 \mathrm{~T}^{*} \mathrm{~T}\right)_{\mathrm{n}^{\prime} \mathrm{n}}\right|
$$

as a measure of the error in our truncated T-matrix. A nice feature of $\operatorname{Err}(\mathrm{N})$ is that it measures errors due to truncation of the T-matrix as well as errors in the computation of the T-matrix entries.

For our numerical experiments, we developed a T-matrix class for the far field and T-matrix computations involved in evaluating $S^{\infty} \widetilde{\boldsymbol{e}}_{\mathrm{n}}$ using the fundamental solution and non-polynomial finite element methods [1]. 
TABLE 1: Convergence of backscattered ACS (in decibels per square metre) and T-matrix for a sound-soft and sound-hard trefoil of diameter $16 \lambda$.

\begin{tabular}{ccccc}
\hline $\mathrm{N}$ & $\begin{array}{c}\text { sound-soft } \partial \mathrm{D} \\
\text { backscattered ACS }\end{array}$ & $\begin{array}{c}\operatorname{Err}(\mathrm{N}) \\
\text { in }(21)\end{array}$ & $\begin{array}{c}\text { sound-hard DD } \\
\text { backscattered ACS }\end{array}$ & $\begin{array}{c}\operatorname{Err}(\mathrm{N}) \\
\text { in }(21)\end{array}$ \\
\hline 58 & -1.0250887 & $6.5 \mathrm{E}-4$ & -0.9453861 & $7.9 \mathrm{E}-4$ \\
63 & -1.0214336 & $1.3 \mathrm{E}-5$ & -0.9560620 & $1.7 \mathrm{E}-5$ \\
68 & -1.0215187 & $1.2 \mathrm{E}-7$ & -0.9558159 & $1.5 \mathrm{E}-7$ \\
73 & -1.0215179 & $1.2 \mathrm{E}-9$ & -0.9558174 & $1.7 \mathrm{E}-9$ \\
\hline
\end{tabular}

TABLE 2: Convergence of backscattered ACS (in decibels per square metre) and T-matrix for an absorbing and penetrable trefoil of diameter $16 \lambda$.

\begin{tabular}{ccccc}
\hline $\mathrm{N}$ & $\begin{array}{c}\text { absorbing } \partial \mathrm{D} \\
\text { backscattered ACS }\end{array}$ & $\begin{array}{c}\operatorname{Err}(\mathrm{N}) \\
\text { in }(21)\end{array}$ & $\begin{array}{c}\text { penetrable } \partial \mathrm{D} \\
\text { backscattered ACS }\end{array}$ & $\begin{array}{c}\operatorname{Err}(\mathrm{N}) \\
\text { in }(21)\end{array}$ \\
\hline 58 & -0.932000 & $8.7 \mathrm{E}-4$ & 0.9369388 & $3.1 \mathrm{E}-4$ \\
63 & -0.943797 & $1.9 \mathrm{E}-5$ & 0.9396784 & $4.7 \mathrm{E}-6$ \\
68 & -0.943520 & $1.6 \mathrm{E}-7$ & 0.9396014 & $3.9 \mathrm{E}-8$ \\
73 & -0.943522 & $1.8 \mathrm{E}-9$ & 0.9396021 & $3.2 \mathrm{E}-9$ \\
\hline
\end{tabular}

TABLE 3: T-matrix computation error and time for various obstacles.

\begin{tabular}{ccccc}
\hline \multicolumn{2}{c}{ Scatterer (type) } & diameter & Err & CPU time \\
\hline trefoil & (sound-soft) & $12 \lambda$ & $1.9 \mathrm{E}-11$ & $0.7 \mathrm{~s}$ \\
trefoil & (sound-soft) & $16 \lambda$ & $1.2 \mathrm{E}-09$ & $3.3 \mathrm{~s}$ \\
trefoil & (sound-hard) & $16 \lambda$ & $1.7 \mathrm{E}-09$ & $3.2 \mathrm{~s}$ \\
trefoil & (absorbing) & $16 \lambda$ & $1.8 \mathrm{E}-09$ & $3.3 \mathrm{~s}$ \\
trefoil & (penetrable) & $16 \lambda$ & $3.2 \mathrm{E}-09$ & $4.3 \mathrm{~s}$ \\
square & (sound-soft) & $12 \lambda$ & $2.6 \mathrm{E}-13$ & $33.4 \mathrm{~s}$ \\
\hline
\end{tabular}


In our experiments, we first compute the truncated $\mathrm{N} \times \mathrm{N}$ T-matrix for various values of $N$. The required $N$ depends on the diameter $a \lambda$ of the scatterer, where $\lambda=2 \pi / k$ is the wavelength of the incident wave and $a$ is a positive constant. Tables 1 and 2 demonstrate increasing accuracy of the T-matrix with respect to $\mathrm{N}$ for a smooth trefoil shaped obstacle (Figures 1 and 2), with diameter $16 \lambda$ and various properties, by tabulating $\operatorname{Err}(\mathrm{N})$ in (21) and also the backscattered acoustic cross section (ACS), computed using the T-matrix, for an incident wave $\mathfrak{u}^{\mathrm{i}}(\boldsymbol{x})=\exp (\mathfrak{i k} \boldsymbol{x} \cdot \widehat{\mathbf{d}})$ where $\widehat{\mathbf{d}}=$ $(\sqrt{3} / 2,1 / 2)$. Increasing accuracy in the backscattered ACS is indicated by the increasing number of matching digits as $\mathrm{N}$ increases.

Table 3 summarises the CPU time required to compute the T-matrix, in Matlab on a $2.26 \mathrm{GHz}$ laptop, and error in the T-matrix for the trefoil and a square scatterer. Figures 1 and 2 plot their monostatic ACS.

Acknowledgments Valuable discussions with Dr A. H. Barnett and Dr T. Betcke, and the support of EPSRC Grant EP/F067798/1 (for the first author's research time at University of Reading) are gratefully acknowledged.

\section{References}

[1] A. H. Barnett and T. Betcke. An exponentially convergent nonpolynomial finite element method for time-harmonic scattering from polygons. SIAM J. Sci. Comput., 2010, to appear. http: //www . personal.reading. ac.uk/ sp900945/papers/BaBe09.pdf. C217, C222, C225

[2] D. Colton and R. Kress. Inverse Acoustic and Electromagnetic Scattering Theory. Springer, 1998. C222 


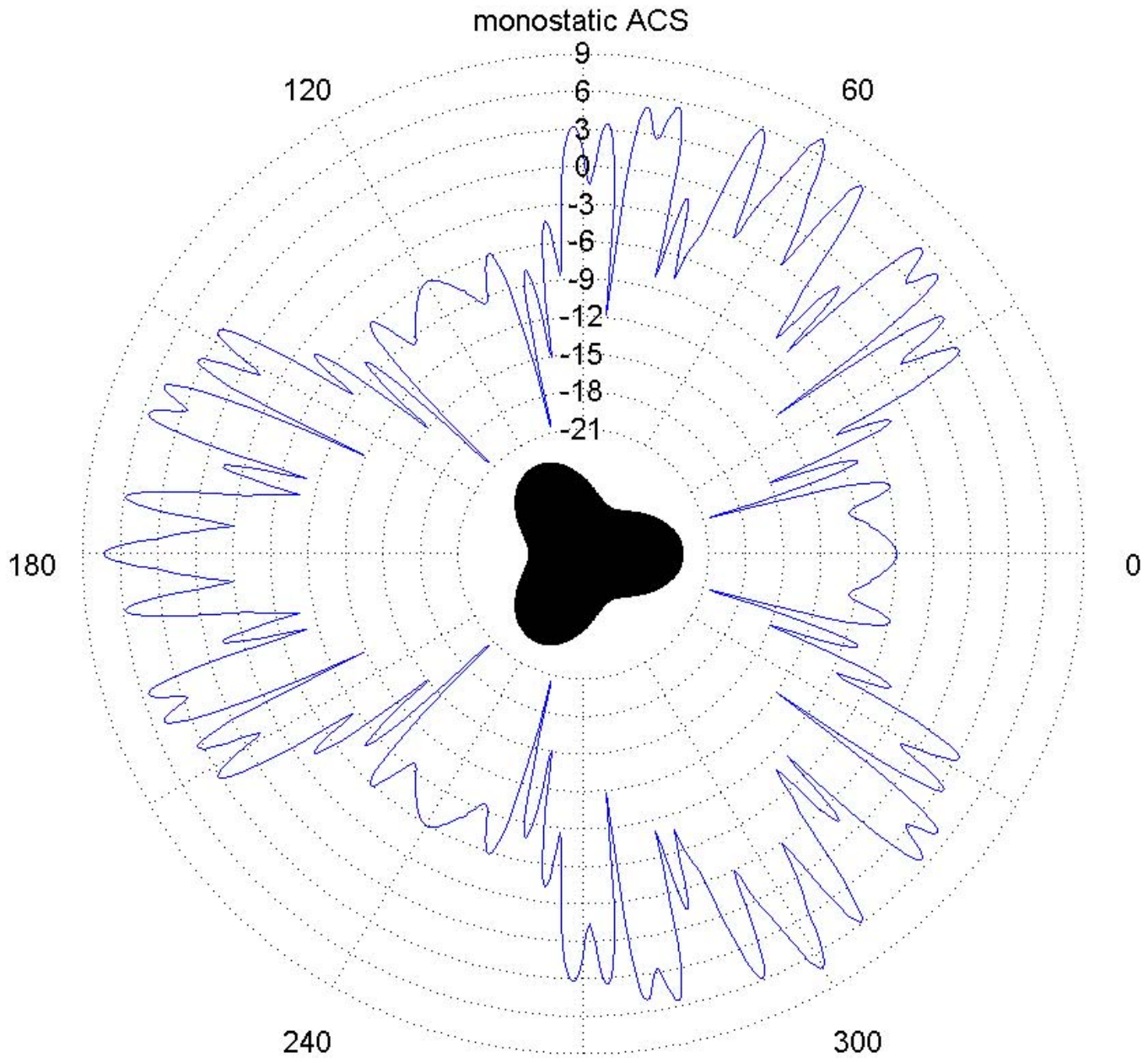

Figure 1: T-matrix based simulation of monostatic ACS (in decibels per square metre, with 1000 incident waves) of a smooth penetrable trefoil scatterer of diameter $16 \lambda$ (with refractive index 0.5 ). 


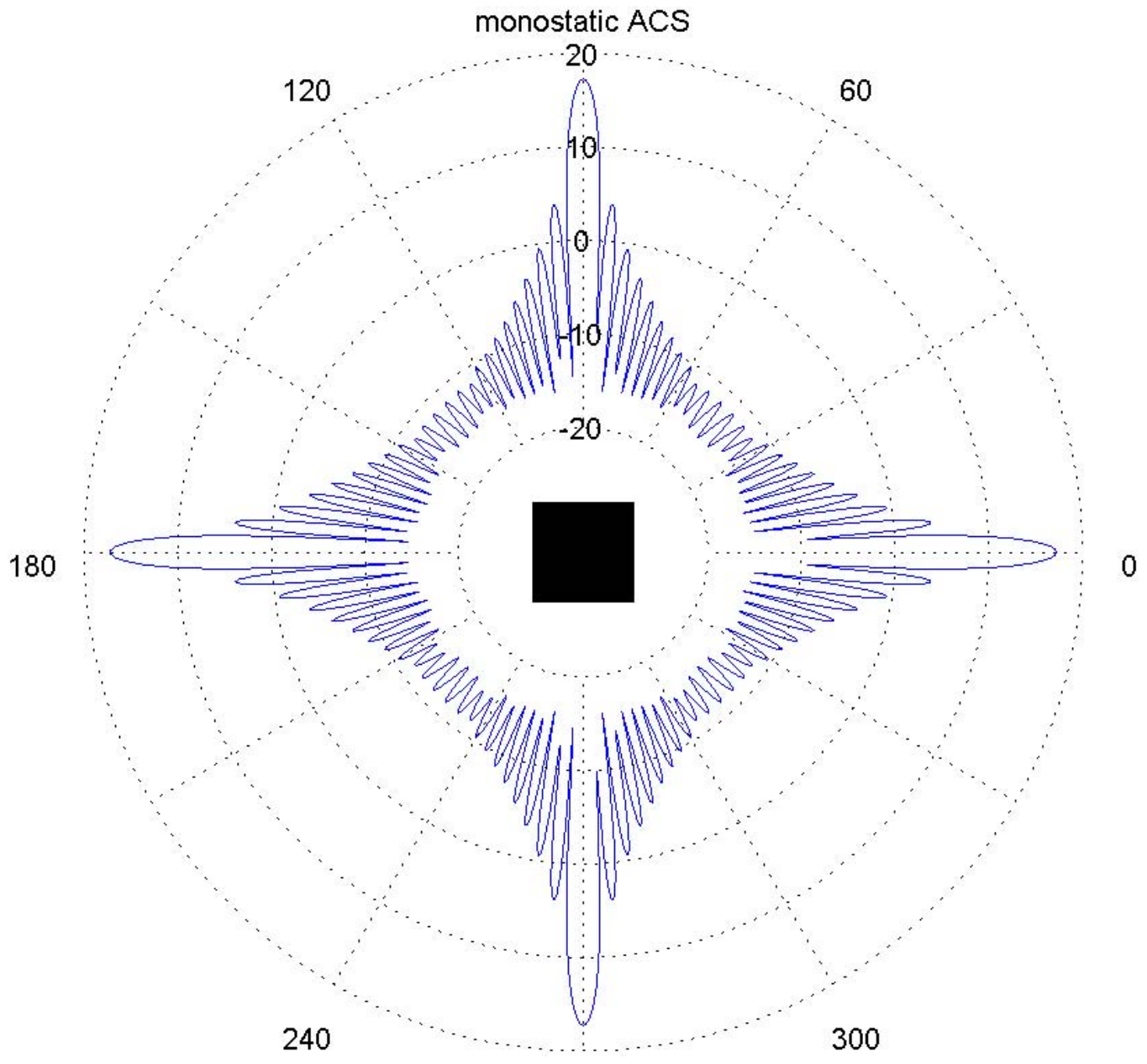

Figure 2: T-matrix based simulation of monostatic ACS (in decibels per square metre, with 1000 incident waves) of a non-smooth sound-soft scatterer of diameter $12 \lambda$. 
[3] A. Doicu, T. Wriedt, and Y. Eremin. Light Scattering by Systems of Particles. Null-Field Method with Discrete Sources - Theory and Programs. Springer Verlag, 2006. C216

[4] M. Ganesh and S. C. Hawkins. A far-field based T-matrix method for three dimensional acoustic scattering. ANZIAM J., 50:C121-C136, 2008. http://anziamj . austms.org.au/ojs/index.php/ANZIAMJ/ article/view/1441 C217

[5] M. Ganesh and S. C. Hawkins. Three dimensional electromagnetic scattering T-matrix computations. J. Comput. Appl. Math., 2010. doi:10.1016/j.cam.2009.08.018. C217

[6] P. A. Martin. Multiple Scattering: Interaction of Time-Harmonic Waves with $N$ Obstacles. Cambridge University Press, 2006. C216, C217, C222

[7] M. I. Mishchenko, L. D. Travis, and A. A. Lacis. Multiple Scattering of Light by Particles: Radiative Transfer and Coherent Backscattering. Cambridge University Press, 2006. C216

[8] P.C. Waterman. Matrix formulation of electromagnetic scattering. Proc. IEEE, 53:805-812, 1965. C216

\section{Author addresses}

1. M. Ganesh, Department of Mathematical and Computer Sciences, Colorado School of Mines, Golden, CO 80401, USA. mailto:mganesh@mines . edu

2. S. C. Hawkins, Department of Mathematics, Macquarie University, Sydney, NSW 2109, Australia.

mailto: shawkins@maths . mq. edu . au 